PROCEEDINGS OF THE

AMERICAN MATHEMATICAL SOCIETY

Volume 126, Number 3, March 1998, Pages 835-840

S 0002-9939(98)04084-2

\title{
ON THE INTERIOR DERIVATIVE BLOW-UP FOR THE CURVATURE EVOLUTION OF CAPILLARY SURFACES
}

\author{
KEISUI ASAI AND NAOYUKI ISHIMURA \\ (Communicated by Jeffrey B. Rauch)
}

\begin{abstract}
We give examples of the interior derivative blow-up solutions for the curvature evolution of capillary surfaces over a bounded domain in $\mathbf{R}^{N}$.
\end{abstract}

\section{INTRODUCTION}

Let $\Omega \subset \mathbf{R}^{N}$ be a bounded domain with boundary of class $C^{k}(k \geq 2)$. We are concerned with the problem:

$$
\begin{aligned}
\frac{1}{\sqrt{1+|D u|^{2}} \frac{\partial u}{\partial t}-\operatorname{div}\left(\frac{D u}{\sqrt{1+|D u|^{2}}}\right)-\kappa u=0} & \text { in } \Omega \times(0, T), \\
u=0 & \text { on } \partial \Omega \times(0, T), \\
u(x, 0)=u_{0}(x) \geq 0 & \text { on } \Omega,
\end{aligned}
$$

where $D u$ denotes the gradient of $u$ and $\kappa>0$ a positive constant. $u_{0}$ is a continuous function with compact support, which will be taken in a class defined later.

The equation (1) originates in the curvature evolution for capillary surfaces. In fact, one can express (1) in the form

$$
v=H+\kappa u,
$$

where $v$ means the normal velocity and $H$ the mean curvature. The force term $\kappa u$ will be legitimately called a capillary term with a capillarity constant $\kappa$ (see [5]). The topic of curvature evolution has attracted many researchers and much progress has been made. However, only a little attention seems to be paid to the analysis of (4). We refer to [8], [12].

Our interest is in the interior derivative blow-up, which is motivated by a recent work of Y. Giga [6]. He discussed a one-dimensional parabolic equation

$$
\begin{aligned}
u_{t}-a\left(u_{x}\right) u_{x x}-f\left(u_{x}\right) g\left(u_{x}\right) & =0 & & \text { in } \Omega \times(0, T), \\
u & =0 & & \text { on } \partial \Omega \times(0, T), \\
u(x, 0) & =u_{0} & & \text { in } \Omega,
\end{aligned}
$$

and constructed derivative blow-up solutions. By derivative blow-up, we mean that the gradient of $u$ goes to infinity as $t \rightarrow T$ but $\sup _{\Omega}|u|$ stays bounded. Here $\Omega$ is an

Received by the editors March 28, 1996 and, in revised form, September 10, 1996.

1991 Mathematics Subject Classification. Primary 35B40, 35K55, 58G11.

Key words and phrases. Derivative blow-up, curvature evolution, capillary surfaces. 
open interval and $a>0, f \geq 0, g$ are $C^{1}$-class functions on $\mathbf{R}$ assumed to satisfy some growth relations. Typical examples include

$$
a(p)=1 /\left(1+p^{2}\right)^{\alpha}, \quad g(p)=\left(1+p^{2}\right)^{1 / 2}, \quad \alpha \geq 1 / 2,
$$

and $f$ is globally Lipschitz.

One of the authors extends [6] to the radially symmetric case of (1) and shows examples of similar derivative blow-ups [2]. In the present paper, on the other hand, we remove the restriction of radial symmetry from [2] and present interior derivative blow-up solutions. Our main result is the theorem in the next section.

The method of construction we employ as well as in [2] is based on the idea of [6], which relies on a geometric argument. We deform a surface of constant mean curvature self-similarly in time so as to formulate sub- and supersolutions. The same consideration is applied to estimate the boundary gradient; the distance function from the boundary is involved. To our knowledge, this type of technique to give boundary gradient estimates by use of the distance function seems to be new.

The derivative blow-up phenomenon is now going under investigation by several authors. Most researches, however, seem to be focused on the boundary gradient blow-up. Moreover, known results of the interior derivative blow-up are essentially one-dimensional. In this respect, we refer to a recent work by S.B. Angenent and M. Fila [1]. For other studies, see [3], [4], [9], [10] and the references therein.

\section{INTERIOR DERIVATIVE BLOW-UP SOLUTIONS}

We begin with introducing some notations to state our result clearly.

Let $B_{R} \subset \Omega$ be the biggest ball contained in $\Omega$ and take a coordinate system so that the zero is the origin of $B_{R}$. Since $\partial \Omega$ is $C^{k}$ with $k \geq 2$, the distance function $d(x):=\operatorname{dist}(x, \partial \Omega)$ is defined and $C^{k}$ on $\Gamma_{\mu}:=\{x \in \Omega \mid \bar{d}(x) \leq \mu\}$, where $\mu>0$ is sufficiently small. See $[7, \S 14.6]$ or [11]. Choose $m>6 / R$ so large that $|\triangle d| / m \leq \frac{1}{2}$ on $\Gamma_{1 / m}$.

Fix positive constants $M, n$ with the condition

$$
M>5 m N+n, \quad 1 \gg n+\frac{n}{\kappa}, \quad 2 m n>\kappa,
$$

taking $m$ larger if necessary, and consider the next ordinary differential equations:

$$
\begin{array}{ll}
\lambda_{1}^{\prime}(t)=m\left(M-\frac{m N}{\lambda_{1}(t)}\right), & \lambda_{1}(0)=1, \\
\lambda_{2}^{\prime}(t)=-m\left(n+\frac{2 m N}{\lambda_{2}(t)}\right), & \lambda_{2}(0)=1 .
\end{array}
$$

The solution $\lambda_{1}(t)$ of (5) exists for all $t \geq 0$ with $\lambda_{1}^{\prime}(t)>0$. We define $t_{0}=t_{0}(m, M)$ so that $\lambda_{1}(t) \leq 2$ on $0 \leq t \leq t_{0}$. The solution $\lambda_{2}(t)$ of $(6)$ exists for $0 \leq t<\tau_{0}$ with $\lambda_{2}^{\prime}(t)<0$, where $\tau_{0}$ is the maximal existence time. We define $\tau_{1}=\tau_{1}(m)$ so that $\lambda_{2}(t) \geq 1 / 2$ on $0 \leq t \leq \tau_{1}$. Set $t_{1}:=\min \left\{t_{0}, \tau_{1}\right\}$.

For later purposes, we introduce domains

$$
Q_{\tau, a}^{i}:=\left\{(x, t) \in \Omega \times(0, \tau)|||x|-a \mid<\frac{\lambda_{i}(t)}{m}\right\}
$$

for $i=1,2$ and

$$
Q_{\tau, 1 / 2 m}^{\Gamma}:=\left\{(x, t) \in \Omega \times(0, \tau)|| d(x)-\frac{1}{2 m} \mid<\frac{\lambda_{2}(t)}{m}\right\} .
$$


Furthermore, we write

$$
v(|x|)=\sqrt{1-|x|^{2}} .
$$

Now we specialize a class of functions for the initial values, which is essentially developed in [6] and referred to as a class of functions with a sharply decreasing part.

Definition. A positive function $u_{0}$ on $\Omega$ is said to be in the class (SDP) if the following conditions are satisfied:

(i) $u_{0}(x)>\frac{v(m|x|)}{m}+\frac{M}{\kappa}$ on $\left\{|x|<\frac{1}{m}\right\}$;

(ii) $u_{0}(x)<-\frac{v(m(|x|-a))}{m}+\frac{n}{\kappa}$ on $\left\{a-\frac{1}{m}<|x|<a+\frac{1}{m}\right\}$;

(iii) $a=\frac{2}{m}+\delta$ with $0<\delta<\min \left\{t_{1} L, \frac{1}{m}\right\}$, where $L:=M-5 m N-n>0$;

(iv) $u_{0}(x)=0$ on $\left\{x \in \Omega|| x \mid \geq R-\frac{2}{m}\right\}$.

We remark that $u_{0}(x)$ need not be radially symmetric.

Now we state our main result.

Theorem. Suppose $u_{0}$ be in the class (SDP). Then the maximal existence time $T_{0}$ of the solution $u$ of (1), (2), (3) is bounded by $\delta / L$. Moreover we have

$$
\sup _{\Omega \times\left(0, T_{0}\right)}|u|<\infty, \quad \sup _{\partial \Omega \times\left(0, T_{0}\right)}|D u|<\infty
$$

but

$$
\limsup _{t \rightarrow T_{0}} \sup _{\Omega}|D u|=\infty .
$$

Proof. The proof is divided into three steps.

1. $T_{0}<\delta / L$.

Introduce comparison functions

$$
u^{-}(x, t):=\frac{\lambda_{1}(t)}{m} v\left(\frac{m}{\lambda_{1}(t)}|x|\right)+\frac{M}{\kappa}
$$

on $Q_{t_{0}, 0}^{1}$ and

$$
u^{+}(x, t):=-\frac{\lambda_{2}(t)}{m} v\left(\frac{m}{\lambda_{2}(t)}(|x|-a)\right)+\frac{n}{\kappa}
$$

on $Q_{\tau_{1}, a}^{2}$. Recalling $v(|x|)=\sqrt{1-|x|^{2}}$, we compute

$$
\begin{aligned}
F\left(u^{-}\right) & :=\frac{\left(u^{-}\right)_{t}}{\sqrt{1+\left|D u^{-}\right|^{2}}}-\operatorname{div}\left(\frac{D u^{-}}{\sqrt{1+\left|D u^{-}\right|^{2}}}\right) \\
& =\frac{1}{m} \lambda_{1}^{\prime}+\frac{m N}{\lambda_{1}}=M \quad \text { on } Q_{t_{0}, 0}^{1}, \\
F\left(u^{+}\right) & =-\frac{1}{m} \lambda_{2}^{\prime}-\frac{m}{\lambda_{2}}\left(N-a \frac{N-1}{|x|}\right) \\
& >-\frac{1}{m} \lambda_{2}^{\prime}-\frac{m}{\lambda_{2}}\left(N-a \frac{N-1}{a+\lambda_{2} / m}\right) \\
& >-\frac{1}{m} \lambda_{2}^{\prime}-\frac{2 m N}{\lambda_{2}}=n \quad \text { on } Q_{\tau_{1}, a}^{2},
\end{aligned}
$$

taking into account (5) and (6) respectively. 
We want to establish that $u^{-}<u$ in $Q_{t_{0}, 0}^{1}$ and $u^{+}>u$ in $Q_{\tau_{1}, a}^{2}$, respectively. Consider the first case. The second one proceeds similarly.

Suppose there exists $0<\bar{t}<t_{0}$ such that $\bar{t}:=\sup \left\{t \leq t_{0} \mid u^{-}<u\right.$ in $\left.Q_{t, 0}^{1}\right\}$. Then we discover

$$
F(u)=\kappa u>\kappa u^{-} \geq M=F\left(u^{-}\right)
$$

in $Q_{t, 0}^{1}$ with $t<\bar{t}$. Since $\partial u^{-} / \partial \nu=-\infty$ on $\left\{|x|=\lambda_{1}(t) / m, 0<t \leq \bar{t}\right\}$ where $\nu$ denotes the outer normal and $u_{0}(x)>u^{-}(x, 0)$ on $\{|x|<1 / m\}$ by assumption, we infer that $u^{-}<u$ in $Q_{\bar{t}, 0}^{1}$ by the maximum principle. This is a contradiction with the definition of $\bar{t}$.

Now we examine the distance $D(t)$ between $Q_{t_{0}, 0}^{1}$ and $Q_{\tau_{1}, a}^{2}$. We find

$$
\begin{aligned}
& D(t)=\left(a-\frac{\lambda_{2}(t)}{m}\right)-\frac{\lambda_{1}(t)}{m}, \\
& D(0)=a-\frac{2}{m}=\delta .
\end{aligned}
$$

Moreover, we have for $0<t<t_{1}$,

$$
\begin{aligned}
-D^{\prime}(t) & =\frac{1}{m}\left(\lambda_{2}^{\prime}(t)+\lambda_{1}^{\prime}(t)\right) \\
& \geq-(n+4 m N)+(M-m N)=L,
\end{aligned}
$$

from which we conclude that $D(t)=0$ before $\delta / L$. In view of $\delta / L<t_{1}, T_{0}<\delta / L$ must hold true.

2. $\sup _{\Omega \times\left(0, T_{0}\right)}|u|<\infty$.

Taking a comparison function $\exp \left(\kappa\left(1+\sup _{\Omega}\left|u_{0}\right|\right) t\right)+\sup _{\Omega}\left|u_{0}\right|$, we arrive at the conclusion.

We remark that $u_{0}$ need not be in (SDP); the finiteness of the existence time is essential.

Combining above two claims, we see that the derivative blow-up must occur. See the argument in [6]. We show that it takes place in the interior.

3. $\sup _{\partial \Omega \times\left(0, T_{0}\right)}|D u|<\infty$.

First we want to estimate $u$ from above in $Q_{T_{0}, 1 / 2 m}^{\Gamma}$. To do this, consider a supersolution

$$
\eta(x, t):=-\frac{\lambda_{2}(t)}{m} v\left(\frac{m}{\lambda_{2}(t)}\left(d(x)-\frac{1}{2 m}\right)\right)+\frac{n}{2 \kappa} .
$$

We compute

$$
\begin{aligned}
F(\eta) & =\frac{\eta_{t}}{\sqrt{1+|D \eta|^{2}}}-\operatorname{div}\left(\frac{D \eta}{\sqrt{1+|D \eta|^{2}}}\right) \\
& =-\frac{\lambda_{2}^{\prime}}{m}-\left(\left(d-\frac{1}{2 m}\right) \triangle d+1\right) \frac{m}{\lambda_{2}} \\
& >-\frac{\lambda_{2}^{\prime}}{m}-\frac{2 m N}{\lambda_{2}}=n .
\end{aligned}
$$


By virtue of $\partial \eta / \partial \nu=\infty$ on $\left\{d(x)=\frac{1}{2 m}+\frac{\lambda_{2}}{m}\right\}$ and $\eta>0$ on $\partial \Omega$, we infer that $\eta>u$ in $Q_{T_{0}, 1 / 2 m}^{\Gamma}$. In particular

$$
\sup _{Q_{T_{0}, 1 / 2 m}^{\Gamma}} u \leq \frac{n}{2 \kappa}-\varepsilon
$$

for sufficiently small $\varepsilon$.

Now we complete the estimate $|D u|$ on $\partial \Omega$. Define, for all sufficiently small $\varepsilon$,

$$
\xi_{\varepsilon}(x):=\frac{1}{n} v(n(d(x)+\alpha(\varepsilon)))+\frac{n}{2 \kappa}-\varepsilon .
$$

Here $\alpha(\varepsilon)(<1 / m)$ can be chosen so that

$$
\begin{aligned}
\left.\xi_{\varepsilon}\right|_{\partial \Omega} & =\frac{1}{n} v(n \alpha(\varepsilon))+\frac{n}{2 \kappa}-\varepsilon=0, \\
\left|D \xi_{\varepsilon}\right|_{\partial \Omega} & <\infty \\
\alpha(\varepsilon) & \rightarrow \frac{1}{m} \quad \text { as } \varepsilon \rightarrow 0 .
\end{aligned}
$$

Moreover we have

$$
F\left(\xi_{\varepsilon}\right)=n((d+\alpha) \triangle d+1) \geq \frac{1}{2} n,
$$

in $\left\{d(x)+\alpha(\varepsilon)<\frac{1}{m}\right\}$. The maximum principle implies that

$$
0<u<\xi_{\varepsilon} \text { in }\left\{(x, t) \in \Omega \times\left(0, T_{0}\right) \mid d(x)+\alpha(\varepsilon)<\frac{1}{m}\right\},
$$

and hence we obtain a desired bound

$$
|D u|_{\partial \Omega} \leq\left|D \xi_{\varepsilon}\right|_{\partial \Omega}<\infty
$$

for $0<t<T_{0}$.

\section{ACKNOWLEDGEMENTS}

We would like to thank Professor Yoshikazu Giga for helpful suggestions and Professors Takushiro Ochiai and Masayasu Mimura for encouragement. Thanks also go to the referee for careful reading and various comments. The second author was partially supported by Grant-in-Aid for Scientific Research (No. 07740140), Japan Ministry of Education, Science, Sports and Culture.

\section{REFERENCES}

1. S.B.Angenent and M.Fila, Interior gradient blow-up in a semilinear parabolic equation, Diff. Integral Eqs. 9 (1996), 865-877. MR 97e:35088

2. K.Asai, Interior derivative blow-up for the curvature evolution of capillary surfaces, Thesis, University of Tokyo (1996).

3. T.Dlotko, Examples of parabolic problems with blowing-up derivatives, J. Diff. Eqs. 154 (1991), 226-237. MR 91k:35133

4. M.Fila and G.M.Lieberman, Derivative blow-up and beyond for quasilinear parabolic equations, Diff. Integral Eqs. 7 (1994), 811-821. MR 95c:35121

5. R.Finn, Equilibrium Capillary Surfaces, Springer-Verlag, New-York, 1986. MR 88f:49001

6. Y.Giga, Interior derivative blow-up for quasilinear parabolic equations, Discrete Conti. Dyn. Syst. 1 (1995), 449-461. MR 96i:35065

7. D.Gilbarg and N.S.Trudinger, Elliptic Partial Differential Equations of Second Order, 2nd edition, Springer-Verlag, Berlin, 1983. MR 86c:35035

8. N.Ishimura, Existence of symmetric capillary surfaces via curvature evolution, J. Fac. Sci. Univ. Tokyo Sect. IA 40 (1993), 419-427. MR 94m:35144 
9. N.Kutev, Global solvability and boundary gradient blow up for one dimensional parabolic equations, in "Progress in Partial Differential Equations : Elliptic and Parabolic Problems," Eds. C.Bandle, J.Bemelmans, M.Chipot and M.Grüter, Longman, 1992, pp. 176-181. CMP 93:04

10. N.Kutev, Gradient blow-ups and global solvability after the blow-up time for nonlinear parabolic equations, in "Evolution Equations, Control Theory and Biomathematics," Eds. P.Clément and G.Lumer, Marcel Dekker, New York, 1994, pp. 301-306. MR 94m:35132

11. J.Serrin, The problem of Dirichlet for quasilinear elliptic differential equations with many independent variables, Philos. Trans. Roy. Soc. London Ser. A 264 (1969), 413-496. MR 43:7772

12. A.Stone, Evolutionary existence proofs for the pendent drops and $n$-dimensional catenary problems, Pacific J. Math. 164 (1994), 147-178. MR 95c:35109

System Laboratory, Fujitsu Cooperation, Minama, Chiba 261, Japan

E-mail address: keisui@tokyo.se.fujitsu.co.jp

Department of Mathematics, Hitotsubashi University, Kunitachi, Tokyo 186, Japan

E-mail address: ishimura@math.hit-u.ac.jp 